\title{
Analytical Evaluation of Nonlinear Effects in MC-CDMA Signals
}

\author{
Rui Dinis, Member, IEEE, and Paulo Silva \\ IST, Tech. Univ. of Lisbon, Av. Rovisco Pais, 1049-001 Lisboa, Portugal, email: \{psilva, rdinis\}@ist.utl.pt
}

\begin{abstract}
The MC-CDMA signals (Multicarrier Coded Division Multiple Access) have strong envelope fluctuations which make them very prone to nonlinear effects. These effects can be both intentional (such as the ones inherent to a nonlinear signal processing for reducing the envelope fluctuations) or not (such as the ones inherent to a nonlinear power amplification).

In this paper we present an analytical tool for the performance evaluation of nonlinear effects in MC-CDMA signals which takes advantage of the Gaussian-like behavior of MC-CDMA signals with a large number of subcarriers and employs results on memoryless nonlinear devices with Gaussian inputs so as to characterize statistically the signals at the output of the nonlinear device. This characterization is then used for an analytical computation of the SIR levels (Signal-to-Interference Ratio) and the BER performance (Bit Error Rate).
\end{abstract}

A set of numerical results is presented and discussed, showing the accuracy of the analytical BER performance analysis in the presence of nonlinear effects ${ }^{1}$.

Keywords: MC-CDMA, nonlinear effects, iterative receivers, Gaussian processes.

\section{Introduction}

The MC-CDMA schemes (Multicarrier Coded Division Multiple Access) [1]-[4] combine a CDMA scheme with OFDM modulation (Orthogonal Frequency Division Multiplexing) [5], so as to allow high transmission rates over severe time-dispersive channels without the need of a complex receiver implementation. Since the spreading is made in frequency domain, the time synchronization requirements are much lower than with conventional direct sequence CDMA schemes. Moreover, the diversity effect inherent to the spreading allows good uncoded performances, or for high code rates.

As with other multicarrier schemes, the MC-CDMA signals have strong envelope fluctuations and high PMEPR values (Peak-to-Mean Envelope Power Ratio) which makes them very prone to nonlinear effects. These nonlinear effects can be both intentional (such as the ones inherent to a nonlinear signal processing for reducing the envelope fluctuations, as in [6][9], or the envelope clipping characteristic that results when a nonlinear amplifier is linearized through an ideal pre-distortion

\footnotetext{
${ }^{1}$ This work was partially supported by the FCT project POSI/CPS/46701/2002 - MC-CDMA and the B-BONE project IST2003-507607.
}

[10]) or not (such as the ones inherent to a nonlinear power amplification [11], [12]).

The performance evaluation of nonlinear transmission usually resorts to Monte-Carlo simulations that require a long computation time. For this reason, analytical approaches have been proposed for the performance evaluation of nonlinear effects in OFDM transmission [7]-[9],[13].

In this paper we present an analytical approach for the performance evaluation of nonlinear effects in MC-CDMA signals. For this purpose, we take advantage of the Gaussianlike behavior of MC-CDMA signals with a large number of subcarriers and employ a statistical characterization of Gaussian signals submitted to a bandpass memoryless nonlinear device [9], [14]. This characterization is then used for an analytical computation of the SIR levels (Signal-to-Interference Ratio) and the BER performance (Bit Error Rate) of MCCDMA schemes in the presence of nonlinear effects.

We also consider an enhanced receiver structure, based on the techniques proposed in [15], [16], where an iterative cancelation of nonlinear distortion effects is carried out. This iterative detection procedure allows good performances even in the presence of significant nonlinear distortion effects such as the ones that arise when MC-CDMA transmission with very low envelope fluctuations is intended. We also take advantage of our statistical models for obtaining, in an analytical way, the achievable performance of these iterative receivers.

This paper is organized as follows: the MC-CDMA schemes considered in this paper are described in Sec. II. The analytical characterization of the transmitted signals in the presence of nonlinear effects is made in Sec. III and used in Sec. IV for performance evaluation purposes. Sec. V describes an enhanced receiver structure with iterative cancelation of nonlinear distortion effects introduced at the transmitter side. A set of performance results is presented in Sec. VI and, finally, Sec. VII is concerned with the conclusions and final remarks to this paper.

\section{Systems Description}

In this paper we consider downlink transmission (i.e., the transmission from the BS (Base Station) to the MT (Mobile Terminal)) within MC-CDMA systems employing frequencydomain spreading, although our approach could also be employed in the uplink transmission. A constant spreading factor $K$ is assumed for all users (the extension to VSF schemes (Variable Spreading Factor) [17] is straightforward). The frequency-domain block to be transmitted by the BS is an 
interleaved version of the block $\left\{S_{k} ; k=0,1, \ldots, N-1\right\}^{2}$, where $N=K M$, with $K$ denoting the spreading factor and $M$ the number of data symbols for each user (see fig. 1(A)). The frequency-domain symbols are given by $S_{k}=$ $\sum_{p=1}^{U} \xi_{p} S_{k, p}$, where $\xi_{p}$ is an appropriate weighting coefficient that accounts for the power control in the downlink (the power associated to the $p$ th user is proportional to $\left|\xi_{p}\right|^{2}$ ) and $S_{k, p}=C_{k M o d K, p} A_{\lfloor k / K\rfloor, p}$ is the $k$ th chip for the $p$ th user $(\lfloor x\rfloor$ denotes 'largest integer not greater than $x$ '), where $\left\{A_{k, p} ; k=\right.$ $0,1, \ldots, M-1\}$ is the block of data symbols associated to the $p$ th user and $\left\{C_{k, p} ; k=0,1, \ldots, K-1\right\}$ is the corresponding spreading sequence. An orthogonal spreading is assumed throughout this paper, with $C_{k, p}$ belonging to a QPSK constellation (Quadrature Phase Shift Keying). Without loss of generality, it is assumed that $\left|C_{k, p}\right|=1$.

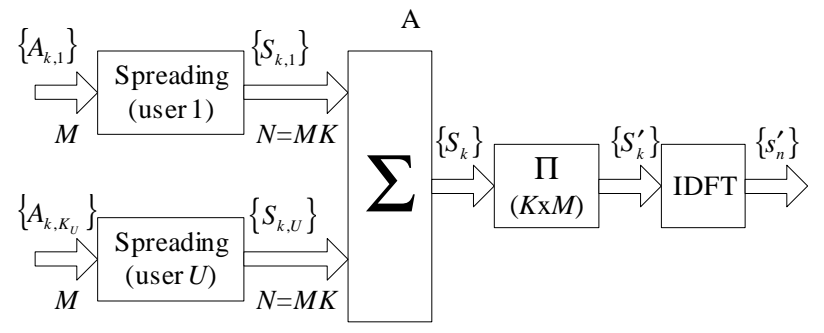

B

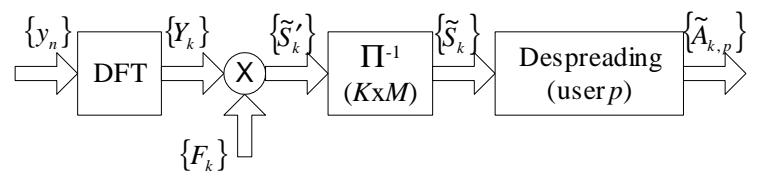

Fig. 1. Conventional MC-CDMA transmitter (A) and receiver (B) for the downlink ( $\Pi$ and $\Pi^{-1}$ denote appropriate chip-level interleaving and deinterleaving operations, respectively).

As with conventional OFDM, an appropriate cyclic extension is appended to each block transmitted by the BS. At the receiver, the cyclic extension is removed and the received samples are passed to the frequency domain, leading to the block $\left\{Y_{k} ; k=0,1, \ldots, N-1\right\}$.

It can be shown that, when the cyclic extension is longer than the overall channel impulse response, the samples $Y_{k}$ can be written as

$$
Y_{k}=H_{k} S_{k}^{\prime}+N_{k}
$$

(a perfect synchronization is assumed), where $H_{k}$ and $N_{k}$ denote the channel frequency response and the noise term for the $k$ th frequency, respectively. The block $\left\{S_{k}^{\prime} ; k=\right.$ $0,1, \ldots, N-1\}$ is the interleaved version of the block $\left\{S_{k} ; k=0,1, \ldots, N-1\right\}$.

Since the orthogonality between users is lost in frequency selective channels, an FDE (Frequency-Domain Equalizer) is

\footnotetext{
${ }^{2}$ Typically, the transmitted frequency-domain block is generated by submitting the block $\left\{S_{k} ; k=0,1, \ldots, N-1\right\}$ to a rectangular interleaver with dimensions $K \times M$, i.e., the different chips associated to a different data symbol are uniformly spread within the transmission band.
}

required before the despreading operation [4], [18]. This means that the receiver structure can be the one depicted in Fig. 1(B). Under perfect channel estimation, the FDE coefficients are given by $F_{k}=S N R H_{k}^{*} /\left(1+S N R\left|H_{k}\right|^{2}\right)$, with $S N R=$ $E\left[\left|S_{k}\right|^{2}\right] / E\left[\left|N_{k}\right|^{2}\right]$, which corresponds to the minimization of the MMSE (Minimum Mean-Squared Error) in the frequencydomain samples $S_{k}$. After removing the idle subcarriers, the equalized samples $\tilde{S}_{k}^{\prime}=Y_{k} F_{k}$ are deinterleaved, leading to the block $\left\{\tilde{S}_{k} ; k=0,1, \ldots, N-1\right\}$. The data symbols associated to the $p$ th user can be estimated from the despreaded samples

$$
\tilde{A}_{k, p}=\sum_{k^{\prime} \in \Psi_{k}} \tilde{S}_{k^{\prime}} C_{k^{\prime}, p}^{*},
$$

with $\Psi_{k}$ denoting the set of frequencies employed to transmit the $k$ th data symbol of each user (for an $K \times M$ interleaving, the set $\Psi_{k}$ is given by $\left.\Psi_{k}=\{k, k+M, \ldots, k+(K-1) M\}\right)$.

\section{Nonlinear Effects in MC-CDMA Signals}

In the previous section an ideal linear transmitter was assumed. However, the MC-CDMA signal has large envelope fluctuations which makes them very prone to nonlinear effects. In this section we present an appropriate model of the transmitted signals when a nonlinear transmitter is considered. For the sake of simplicity, the transmitter structure depicted in Fig. 2 is assumed, where the nonlinear device $g_{C}(\cdot)$ is modeled as a bandpass memoryless nonlinearity [11] operating on an oversampled version of the MC-CDMA burst, i.e., $s_{n}^{C}=g_{C}\left(\left|s_{n}^{\prime \prime}\right|\right) \exp \left(j \arg \left(s_{n}^{\prime \prime}\right)\right)$ (the corresponding AM-to$\mathrm{AM}$ and AM-to-PM conversions are $\left|g_{C}(\cdot)\right|$ and $\arg \left(g_{C}(\cdot)\right)$, respectively). In fact, adding $N^{\prime}-N$ zeros to the original frequency-domain block (i.e., $N^{\prime}-N$ idle subcarriers), followed by an IDFT operation is equivalent to generate a sampled version of the MC-CDMA signal, with an oversampling factor $M_{T x}=N^{\prime} / N$. An optional frequency-domain filtering procedure, through the set of multiplying coefficients $G_{k}, k=0,1, \ldots, N^{\prime}-1$, can also be considered to reduce the out-of-band radiation levels inherent to the nonlinear operation [7].

The transmitter structure of Fig. 2 is similar to the nonlinear signal processing schemes proposed in [7], [8] for reducing the PMEPR of OFDM signals while maintaining the spectral efficiency of conventional OFDM schemes. This transmitter model could also be employed for evaluating the impact of a nonlinear power amplification: we just have to consider an oversampling factor large enough and omit the frequencydomain filtering subsequent to the nonlinear operation.

When the number of subcarriers is high $(N>>1)$ the time-domain coefficients $s_{n}^{\prime \prime}$ can be approximately regarded as samples of a complex Gaussian process. If $E\left[S_{k}\right]=0$ and $E\left[S_{k} S_{k^{\prime}}^{*}\right]=2 \sigma_{S}^{2} \delta_{k, k^{\prime}}\left(\delta_{k, k^{\prime}}=1\right.$ for $k=k^{\prime}$ and 0 otherwise $)$, with $\sigma_{S}^{2}=\frac{1}{2} E\left[\left|S_{k}\right|^{2}\right]$ ( $E[\cdot]$ denotes "ensemble average"), then it can be easily demonstrated that $E\left[s_{n}^{\prime \prime}\right]=0$ and

$$
\begin{array}{r}
E\left[s_{n}^{\prime \prime} s_{n^{\prime}}^{\prime \prime}\right]=R_{s}\left(n-n^{\prime}\right)= \\
=2 \sigma^{2} \frac{\operatorname{sinc}\left(\left(n-n^{\prime}\right) N / N^{\prime}\right)}{\operatorname{sinc}\left(\left(n-n^{\prime}\right) / N^{\prime}\right)} \exp \left(-\frac{j \pi\left(n-n^{\prime}\right)}{N^{\prime}}\right)
\end{array}
$$




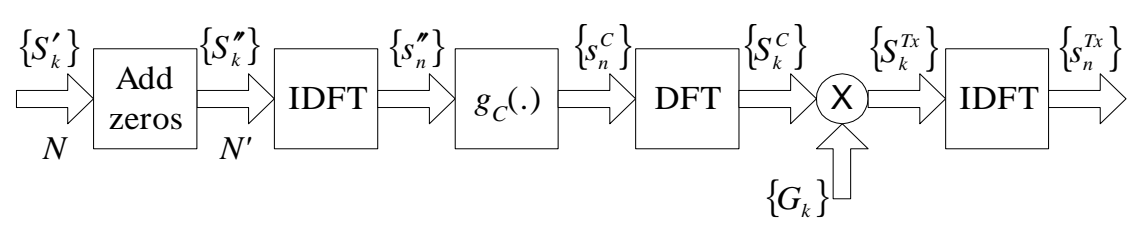

Fig. 2. Transmitter model considered in this paper.

$\left(n, n^{\prime}=0,1, \ldots, N^{\prime}-1\right)$, with $\sigma^{2}=\frac{N}{\left(N^{\prime}\right)^{2}} \sigma_{S}^{2}$.

The output of a memoryless nonlinear device with a Gaussian input can be written as the sum of two uncorrelated components [19]: a useful one, proportional to the input, and a nonlinear self-interference one, i.e.,

$$
s_{n}^{C}=\alpha s_{n}^{\prime \prime}+d_{n},
$$

where $E\left[s_{n}^{\prime \prime} d_{n^{\prime}}^{*}\right]=0$ and $\alpha=E\left[s_{n}^{C} s_{n}^{\prime \prime}{ }^{*}\right] / E\left[\left|s_{n}^{\prime \prime}\right|^{2}\right]=$ $E\left[R g_{C}(R)\right] / E\left[R^{2}\right]$, with $R=\left|s_{n}^{\prime \prime}\right|$. Since $R$ has a Rayleigh distribution, we get

$$
\alpha=\frac{1}{2 \sigma^{2}} \int_{0}^{+\infty} R g_{C}(R) \frac{R}{\sigma^{2}} \exp \left(-\frac{R^{2}}{2 \sigma^{2}}\right) d R .
$$

The average power of the useful component is $P_{N L}^{S}=$ $|\alpha|^{2} \sigma^{2}$, and the average power of the nonlinear selfinterference component is given by $P_{N L}^{I}=P_{N L}-P_{N L}^{S}$, where $P_{N L}$ denotes the average power of the signal at the nonlinearity output, given by

$$
\begin{gathered}
P_{N L}=\frac{1}{2} E\left[\left|s_{n}^{C}\right|^{2}\right]=\frac{1}{2} E\left[\left|g_{C}(R)\right|^{2}\right]= \\
=\frac{1}{2} \int_{0}^{+\infty}\left|g_{C}(R)\right|^{2} \frac{R}{\sigma^{2}} \exp \left(-\frac{R^{2}}{2 \sigma^{2}}\right) d R .
\end{gathered}
$$

It can be shown [14] that

$$
=\begin{array}{r}
E\left[s_{n}^{C} s_{n^{\prime}}^{C *}\right]=R_{s}^{C}\left(n-n^{\prime}\right)= \\
\sum_{\gamma=0}^{+\infty} 2 P_{2 \gamma+1} \frac{\left(R_{s}\left(n-n^{\prime}\right)\right)^{\gamma+1}\left(R_{s}^{*}\left(n-n^{\prime}\right)\right)^{\gamma}}{\left(R_{s}(0)\right)^{2 \gamma+1}},
\end{array}
$$

where the coefficient $P_{2 \gamma+1}$ denotes the total power associated to the IMP (Inter-Modulation Product) of order $2 \gamma+1$. This coefficient can be obtained as follows [20]:

$$
P_{2 \gamma+1}=\frac{\left|v_{2 \gamma+1}\right|^{2}}{2 \gamma !(\gamma+1) !},
$$

where

$$
v_{2 \gamma+1}=\int_{0}^{+\infty} R g_{C}(R) W_{2 \gamma+1}\left(\frac{R}{\sqrt{2 \sigma^{2}}}\right) d R,
$$

with

$$
W_{2 \gamma+1}(x)=\frac{\gamma !}{2} e^{-x^{2}} x L_{\gamma}^{(1)}\left(x^{2}\right)
$$

$\left(L_{\gamma}^{(1)}(\cdot)\right.$ denotes a generalized Laguerre polynomial of order $\gamma$ [21]).
Since $R_{s}^{C}\left(n-n^{\prime}\right)=|\alpha|^{2} R_{s}\left(n-n^{\prime}\right)+E\left[d_{n} d_{n^{\prime}}^{*}\right]$, it can be easily recognized that $P_{1}=|\alpha|^{2} \sigma^{2}$ and

$$
\begin{aligned}
& E\left[d_{n} d_{n^{\prime}}^{*}\right]=R_{d}\left(n-n^{\prime}\right)= \\
& =\sum_{\gamma=1}^{+\infty} 2 P_{2 \gamma+1} \frac{\left(R_{s}\left(n-n^{\prime}\right)\right)^{\gamma+1}\left(R_{s}^{*}\left(n-n^{\prime}\right)\right)^{\gamma}}{\left(R_{s}(0)\right)^{2 \gamma+1}} \text {. }
\end{aligned}
$$

The total self-interference power is

$$
P_{N L}^{I}=\frac{1}{2} R_{d}(0)=\sum_{\gamma=1}^{+\infty} P_{2 \gamma+1}=P_{N L}-P_{N L}^{S} .
$$

Having in mind (4) and the signal processing chain in Fig. 2, the frequency-domain block $\left\{S_{k}^{T x}=S_{k}^{C} G_{k} ; k=\right.$ $\left.0,1, \ldots, N^{\prime}-1\right\}$ can obviously be decomposed into useful and nonlinear self-interference components:

$$
S_{k}^{T x}=\alpha S_{k}^{\prime \prime} G_{k}+D_{k} G_{k},
$$

where $\left\{D_{k} ; k=0,1, \ldots, N^{\prime}-1\right\}$ is the DFT of $\left\{d_{n} ; n=\right.$ $\left.0,1, \ldots, N^{\prime}-1\right\}$. Clearly, $E\left[D_{k}\right]=0$ and

$$
E\left[D_{k} D_{k^{\prime}}^{*}\right]= \begin{cases}N^{\prime} G_{d}(k), & k=k^{\prime} \\ 0, & \text { otherwise }\end{cases}
$$

$\left(k, k^{\prime}=0,1, \ldots, N^{\prime}-1\right)$, where $\left\{G_{d}(k) ; k=0,1, \ldots, N^{\prime}-\right.$ $1\}$ denotes the DFT of the block $\left\{R_{d}(n) ; n=0,1, \ldots, N^{\prime}-1\right\}$ $\left(R_{d}\left(n-n^{\prime}\right)=E\left[d_{n} d_{n^{\prime}}^{*}\right]\right)$. Moreover, $D_{k}$ exhibits quasiGaussian characteristics for any $k$, provided that the number of subcarriers is high enough. Clearly, $E\left[S_{k}^{C} S_{k^{\prime}}^{C *}\right]=0$ for $k \neq k^{\prime}$ and $E\left[\left|S_{k}^{C}\right|^{2}\right]=N^{\prime} G_{s}^{C}(k)$, where $\left\{G_{s}^{C}(k)=\right.$ $\left.|\alpha|^{2} G_{s}(k)+G_{d}(k) ; k=0,1, \ldots, N^{\prime}-1\right\}$ denotes the DFT of $\left\{R_{s}^{C}(n) ; k=0,1, \ldots, N^{\prime}-1\right\}$ (given by (7)), with $\left\{G_{s}(k) ; k=0,1, \ldots, N^{\prime}-1\right\}=\operatorname{DFT}\left\{R_{s}(n) ; n=\right.$ $\left.0,1, \ldots, N^{\prime}-1\right\}$. Therefore, $E\left[S_{k}^{T x} S_{k^{\prime}}^{T x *}\right]=0$ for $k \neq k^{\prime}$, and $E\left[\left|S_{k}^{T x}\right|^{2}\right]=\left|G_{k}\right|^{2} E\left[\left|S_{k}^{C}\right|^{2}\right]=N^{\prime}\left|G_{k}\right|^{2} G_{s}^{C}(k)$.

Clearly, the power of the useful and nonlinear selfinterference components of the transmitted signals are

$$
P_{T x}^{S}=\sum_{k} E\left[\left|\alpha S_{k}^{\prime \prime} G_{k}\right|^{2}\right]
$$

and

$$
P_{T x}^{I}=\sum_{k} E\left[\left|D_{k} G_{k}\right|^{2}\right],
$$

respectively. We can also define the power of the nonlinear self-interference component in the in-band region as

$$
P_{T x, I B}^{I}=\sum_{k \text { in-band }} E\left[\left|D_{k} G_{k}\right|^{2}\right]
$$


When $G_{k}=1$ for the $N$ in-band subcarriers, $P_{T x}^{S}=P_{N L}^{S}$. If we also have $G_{k}=0$ for the $N^{\prime}-N$ out-of-band subcarriers then $P_{T x, I B}^{I}=P_{T x}^{I}$.

The "signal-to-interference ratio" (SIR) for the transmitted signals is

$$
S I R_{T x}=\frac{P_{T x}^{S}}{P_{T x}^{I}} \leq S I R_{N L}=\frac{P_{N L}^{S}}{P_{N L}^{I}},
$$

where $S I R_{N L}$ denotes the SIR at the output of the nonlinear device; the SIR for the in-band region is

$$
S I R_{T x, I B}=\frac{P_{T x}^{S}}{P_{T x, I B}^{I}} .
$$

We can also define a SIR for each subcarrier, given by

$$
S I R_{k}=\frac{E\left[\left|\alpha S_{k}^{\prime \prime}\right|^{2}\right]}{E\left[\left|D_{k}\right|^{2}\right]} .
$$

Without oversampling, (3) leads to $R_{s}\left(n-n^{\prime}\right)=2 \sigma^{2} \delta_{n, n^{\prime}}$ and, from (7),

$$
R_{s}^{C}\left(n-n^{\prime}\right)= \begin{cases}2 \sum_{\gamma=0}^{+\infty} P_{2 \gamma+1}, & k=k^{\prime} \\ 0, & \text { otherwise. }\end{cases}
$$

Therefore,

$$
S I R_{k}=\frac{P_{1}}{\sum_{\gamma=1}^{+\infty} P_{2 \gamma+1}}=S I R_{N L}=S I R_{T x}=S I R_{T x, I B},
$$

which is independent of $k$, when $M_{T x}=1$. For $M_{T x}>1$ (i.e., when $\left.N^{\prime}>N\right), R_{s}\left(n-n^{\prime}\right) \neq 2 \sigma^{2} \delta_{n, n^{\prime}}$ and $S I R_{k}$ is a function of $k$, since $E\left[\left|D_{k}\right|^{2}\right]$ depends also on $k$.

\section{Analytical Performance Evaluation}

\section{A. SIR Levels and BER Performance}

From (1), the frequency-domain samples at the receiver are given by $Y_{k}=S_{k}^{T x} H_{k}+N_{k}$, provided that the guard interval is long enough.

For an ideal Gaussian channel, the detection of the $k$ th symbol transmitted by the $p$ th user is based on the "despreaded" samples

$$
\tilde{A}_{k, p}=\sum_{k^{\prime} \in \Psi_{k}} Y_{k^{\prime}} C_{k^{\prime}, p}^{*}=\alpha \xi_{p} K A_{k, p}+D_{k, p}^{e q}+N_{k, p}^{e q}
$$

(see (2)). As referred above, for a frequency-selective channel we need to perform an FDE previous to the "despreading" operation. In (23), $D_{k, p}^{e q}=\sum_{k^{\prime} \in \Psi_{k}} D_{k^{\prime}} C_{k^{\prime}, p}^{*}$ and $N_{k, p}^{e q}=\sum_{k^{\prime} \in \Psi_{k}} N_{k^{\prime}, p} C_{k^{\prime}, p}^{*}$ denote the equivalent nonlinear self-interference and noise terms for detection purposes, respectively.

Clearly, the power of the nonlinear self-interference term, $D_{k, p}^{e q}$, is

$$
P_{k, p}^{I, e q}=\sum_{k^{\prime} \in \Psi_{k}} E\left[\left|D_{k^{\prime}}\right|^{2}\right]=\frac{2 P_{T x, I B}^{I}}{M} \zeta_{k}
$$

with

$$
\zeta_{k}=\frac{M \sum_{k^{\prime} \in \Psi_{k}} E\left[\left|D_{k^{\prime}}\right|^{2}\right]}{\sum_{k^{\prime} \text { in-band }} E\left[\left|D_{k^{\prime}}\right|^{2}\right]}
$$

(it is assumed that $\left|C_{k, p}\right|=1$ ). Since we are employing orthogonal spreading sequences, the "useful" component for detection purposes of the $p$ th user is

$\alpha \sum_{k^{\prime} \in \Psi_{k}} S_{k^{\prime}} C_{k^{\prime}, p}^{*}=\alpha \xi_{p} A_{k, p} \sum_{k^{\prime} \in \Psi_{k}}\left|C_{k^{\prime}, p}\right|^{2}=\alpha \xi_{p} K A_{k, p}$.

By assuming $E\left[\left|A_{k, p}\right|^{2}\right]=1$, the power of the "useful" component for detection purposes of the $p$ th user is

$$
\begin{aligned}
P_{k, p}^{S, e q} & =\left|K \alpha \xi_{p}\right|^{2}=\frac{K\left|\alpha \xi_{p}\right|^{2}}{\sum_{p^{\prime}} \xi_{p^{\prime}}^{2}} \sum_{k^{\prime} \in \Psi_{k}} E\left[\left|S_{k^{\prime}}\right|^{2}\right]= \\
& =\frac{K\left|\alpha \xi_{p}\right|^{2}}{U \overline{\xi^{2}}} \sum_{k^{\prime} \in \Psi_{k}} E\left[\left|S_{k^{\prime}}\right|^{2}\right]=\frac{K}{U} \eta_{\xi, p} \frac{2 P_{T x}^{S}}{M}
\end{aligned}
$$

with $\overline{\xi^{2}}=\sum_{p^{\prime}} \xi_{p^{\prime}}^{2} / U$ and $\eta_{\xi, p}=\xi_{p}^{2} / \overline{\xi_{p}^{2}}$.

Therefore, the corresponding signal-to-self-interference ratio for detection purposes is

$$
S I R_{k, p}^{e q}=\frac{P_{k, p}^{S, e q}}{P_{k, p}^{I, e q}}=\frac{K}{U} \eta_{\xi, p} S I R_{T x, I B} \zeta_{k}^{-1}
$$

From (28), it is clear that the equivalent SIR for detection purposes increases when we decrease the number of users, for a given spreading factor $K$. This is a consequence of the samples of the nonlinear self-interference component, $D_{k} C_{k, p}^{*}$, being uncorrelated, contrarily to the useful components. We can also note that the equivalent SIR for detection purposes is not the same for the different users: the users with smaller attributed powers (i.e., the users that are closer to the BS and/or have better propagation conditions) have worse $S I R_{k, p}^{e q}$ levels, and, consequently, a larger performance degradation due to the nonlinear effects.

Since the nonlinear self-interference component is approximately Gaussian-distributed at the subcarrier level [9], $D_{k}^{e q}$ is also approximately Gaussian-distributed, even when the number of users is small. Therefore, if the data symbols are selected from a QPSK constellation under a Gray mapping rule (the extension to other constellations is straightforward), the BER for an ideal Gaussian channel is approximately given by

$$
B E R_{k, p}=Q\left(\sqrt{S N R_{k, p}^{e q}}\right)
$$

where $Q(\cdot)$ denotes the well-known Gaussian error function and $S N R_{k, p}^{e q}$ denotes an equivalent signal-to-noise ratio for the detection of the $k$ th data symbol, for the $p$ th user. This ratio is given by

$$
S N R_{k, p}^{e q}=\frac{P_{k, p}^{S, e q}}{P_{k, p}^{I, e q}+P^{N, e q}},
$$


where $P^{N, e q}=E\left[\left|N_{k}^{e q}\right|^{2}\right]=K E\left[\left|N_{k}\right|^{2}\right]=2 K N_{0}$, with $N_{0}$ denoting the PSD (Power Spectral Density) of the channel noise. It can be shown that

$$
S N R_{k, p}^{e q}=2 \eta_{S} \eta_{k, p}^{I} \frac{E_{b, p}}{N_{0}},
$$

where

$$
E_{b, p}=\frac{P_{T x}^{S}+P_{T x}^{I}}{2 U M} \eta_{\xi, p}
$$

denotes the average bit energy for the $p$ th user. In (31),

$$
\eta_{S}=\frac{P_{T x}^{S}}{P_{T x}^{S}+P_{T x}^{I}}
$$

and

$$
\eta_{k, p}^{I}=\left(1+2 \frac{U}{K \eta_{\xi, p}} \cdot \frac{P_{T x, I B}^{I} \zeta_{k}}{P_{T x}^{S}+P_{T x}^{I}} \cdot \frac{E_{b, p}}{N_{0}}\right)^{-1} .
$$

Clearly, the degradation factor $\eta_{S}$ is associated to the useless power spent in the transmitted nonlinear self-interference; the degradation factor $\eta_{k, p}^{I}$ is due to the fact that the equivalent, quasi-Gaussian nonlinear self-interference $D_{k}^{e q}$ is added to the Gaussian channel noise.

\section{B. Special Cases and Simplified Formulas}

For most cases of interest, the analytical approach for obtaining the SIR levels and the BER performances described above can be simplified with only a very slight decrease in its accuracy.

As it was referred in the previous section, if there is no oversampling before the nonlinear operation (i.e., for $M_{T x}=$ $\left.N^{\prime} / N=1\right)$, then $P_{T x, I B}^{I}=P_{T x}^{I}=P_{N L}^{I}, P_{T x}^{S} / P_{T x}^{I}=$ $S I R_{T x}=S I R_{N L}$ and $\zeta_{k}=1$. Therefore,

$$
\eta_{S}=\frac{S I R_{N L}}{1+S I R_{N L}}
$$

and $\eta_{k, p}^{I}$, which becomes independent of $k$, is given by

$$
\eta_{k, p}^{I}=\left(1+2 \frac{U}{K \eta_{\xi, p}} \frac{1}{1+S I R_{N L}} \frac{E_{b, p}}{N_{0}}\right)^{-1} .
$$

It can easily be verified that when the "chips" associated to a given data block are uniformly spread in the transmission band (i.e., for a rectangular interleaver with size $K \times M$ ) then $\zeta_{k} \approx 1$, provided that the spreading factor is not too low.

Let us assume now that $M_{T x}>1$. To obtain an approximate formula for the SIR levels that does not require the computation of all IMPs, we will assume that the total nonlinear selfinterference power is associated to the IMP of order 3, i.e., $P_{3}=P_{N L}^{I}$ and $P_{2 \gamma+1}=0, \gamma>1$. This is a slightly pessimistic assumption relatively to the in-band self-interference levels, as depicted in Fig. 3, where it is assumed that $g_{C}(R)$ is an ideal envelope clipping, with normalized clipping level $s_{M} / \sigma=1.0$. In that case, we can derive a closed formula for the power distribution of the nonlinear self-interference component, as well as the total in-band self-interference power, as shown in Appendix A. Using these formulas, it is clear that the average power of the nonlinear self-interference component for the $N$ in-band subcarriers is

$\frac{1}{N} \sum_{k \text { in-band }} E\left[\left|D_{k}\right|^{2}\right] \approx \frac{\kappa\left(M_{T x}\right)}{N} \sum_{k} E\left[\left|D_{k}\right|^{2}\right]=\frac{\kappa\left(M_{T x}\right)}{N} 2 P_{N L}^{I}$,

with $\kappa\left(M_{T x}\right)$ given by (49). This means that, for $G_{k}=1$ for the $N$ in-band subcarriers and 0 for the $N^{\prime}-N$ out-of-band subcarriers,

$$
\eta_{S} \approx \frac{S I R_{N L} / \kappa\left(M_{T x}\right)}{1+S I R_{N L} / \kappa\left(M_{T x}\right)}
$$

and $\eta_{k, p}^{I}$ is almost independent of $k$ and given by

$$
\eta_{k, p}^{I}=\left(1+2 \frac{U}{K \eta_{\xi, p}} \frac{1}{1+S I R_{N L} / \kappa\left(M_{T x}\right)} \frac{E_{b, p}}{N_{0}}\right)^{-1} .
$$

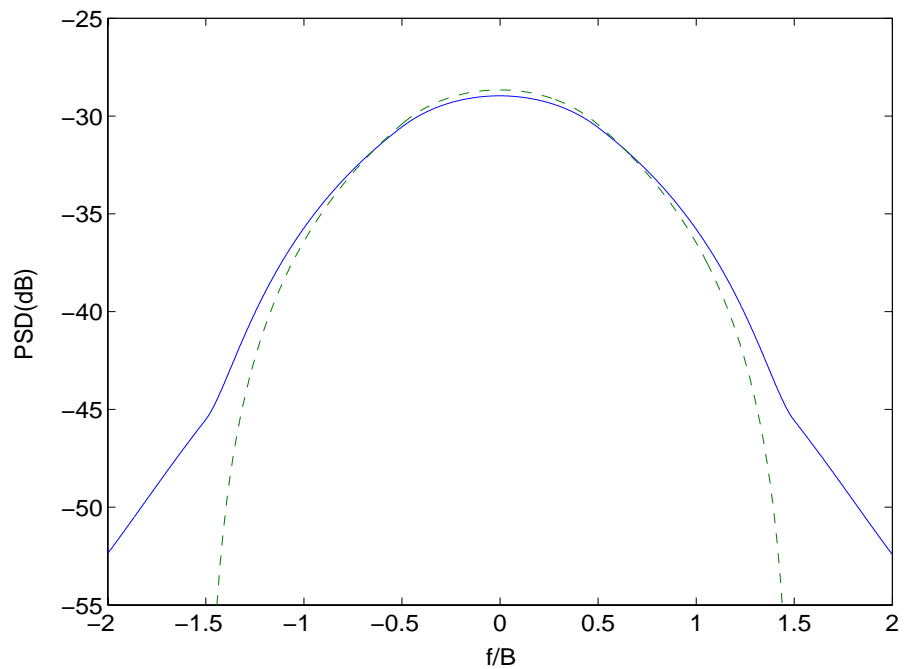

Fig. 3. Evolution of $E\left[\left|D_{k}\right|^{2}\right]$ : exact values (solid line) and approximate values when it is assumed that $P_{3}=P_{N L}^{I}$ and $P_{2 \gamma+1}=0, \gamma>1$ (dashed line).

For $M_{T x} \geq 2, \kappa\left(M_{T x}\right)=2 / 3$ and we have a gain of $3 / 2$ (approximately $1.8 \mathrm{~dB}$ ) in the equivalent SIR levels relatively to the case where there is no oversampling $\left(M_{T x}=1\right)$.

Clearly, the computation of $\eta_{S}$ and $\eta_{k, p}^{I}$ involves only the two integrals inherent to $\alpha$ and $P_{N L}$ (see (5) and (6)). If the nonlinearity corresponds to an ideal envelope clipping, i.e., when

$$
g_{C}(R)= \begin{cases}R, & R \leq s_{M} \\ s_{M}, & R>s_{M}\end{cases}
$$

with $s_{M}$ denoting the clipping level, which is a very common situation, these two integrals can be written in a closed form:

$$
\begin{gathered}
\alpha=1-\exp \left(-\frac{s_{M}^{2}}{2 \sigma^{2}}\right)+\frac{\sqrt{2 \pi} s_{M}}{2 \sigma} Q\left(\frac{s_{M}}{\sigma}\right) \\
P_{N L}=\sigma^{2}\left(1-\exp \left(-\frac{s_{M}^{2}}{2 \sigma^{2}}\right)\right) .
\end{gathered}
$$




\section{Iterative Cancelation of the Deliberate Nonlinear Distortion Effects}

From (13), the received frequency-domain samples can be written as $Y_{k}=\alpha S_{k}^{\prime \prime} H_{k}+D_{k} H_{k}+N_{k}$, for the $N$ "in-band" subcarriers (it is assumed that $G_{k}=1$ for the $N$ in-band subcarriers). It was shown in [15], [16] that we can improve significantly the performance of OFDM schemes submitted to nonlinear devices by employing a receiver with iterative cancelation of nonlinear distortion effects. This concept can be extended to MC-CDMA, leading to the receiver structure of Fig. 4. For each iteration, an estimate $\left\{\hat{S}_{k}^{\prime(i)}\right\}$ of the data vector is obtained from the received vector $\left\{Y_{k}\right\}$ and the distortion vector estimate $\left\{\hat{D}_{k}^{(i-1)}\right\}$ provided by the preceding iteration; next, the distortion vector estimate is updated by using the new data vector estimate (for the first iteration, $\hat{D}_{k}^{(0)}=0$ ). Naturally, this means that we need to jointly detect the data symbols associated to all spreading codes. If a perfect cancelation of the nonlinear self-interference levels is achieved the only degradation is due to the "useless" power spent by the self-interference component and the BER in an ideal AWGN channel is lower-bounded by

$$
B E R_{k, p} \approx Q\left(\sqrt{2 \eta_{S} \frac{E_{b, p}}{N_{0}}}\right),
$$

when the data symbols belong to a QPSK constellation.

\section{Performance Results}

In this paper we present a set of performance results concerning the performance evaluation of nonlinear effects in MC-CDMA transmission. It is assumed that the MC-CDMA signals have a spreading factor $K=64$ and each user has $M=16$ data symbols per block, corresponding to MC-CDMA blocks with length $N=K M=1024$, plus an appropriate cyclic extension. The same power is attributed to each user (i.e., $\xi_{p}=1$ for all users). We consider the transmitter structure depicted in Fig. 2, where an ideal envelope clipping, operating on a sampled version of the MC-CDMA signal, is adopted for reducing the envelope fluctuations of the transmitted signals (unless stated otherwise, an oversampling factor $M_{T x}=2$ is assumed). However, as it was already mentioned, our analytical approach can easily be extended to other nonlinear devices, namely those associated to a nonlinear power amplification (in fact, since we have almost the same SIR levels regardless of the oversampling factor $M_{T x}$, provided that $M_{T x} \geq 2$, our performance results are still valid for a perfectly linearized power amplifier, modeled as an ideal envelope clipping).

Fig. 5 shows the impact of the normalized clipping level $S I R_{T x, I B}$ when $M_{T x}=1$ or 2 . We also include the approximate $S I R_{T x, I B}$ formula that is obtained by using (48), with $M_{T x} \geq 2$ (i.e., $S I R_{T x, I B} \approx 3 S I R_{N L} / 2$ ). From this figure, it is clear that our approximate formula for $S I R_{T x, I B}$ is very accurate, especially for moderate clipping levels. The corresponding values of $\eta_{S}$, depicted in Fig. 6, are close to 1 $(0 \mathrm{~dB})$, unless the clipping level is very low.

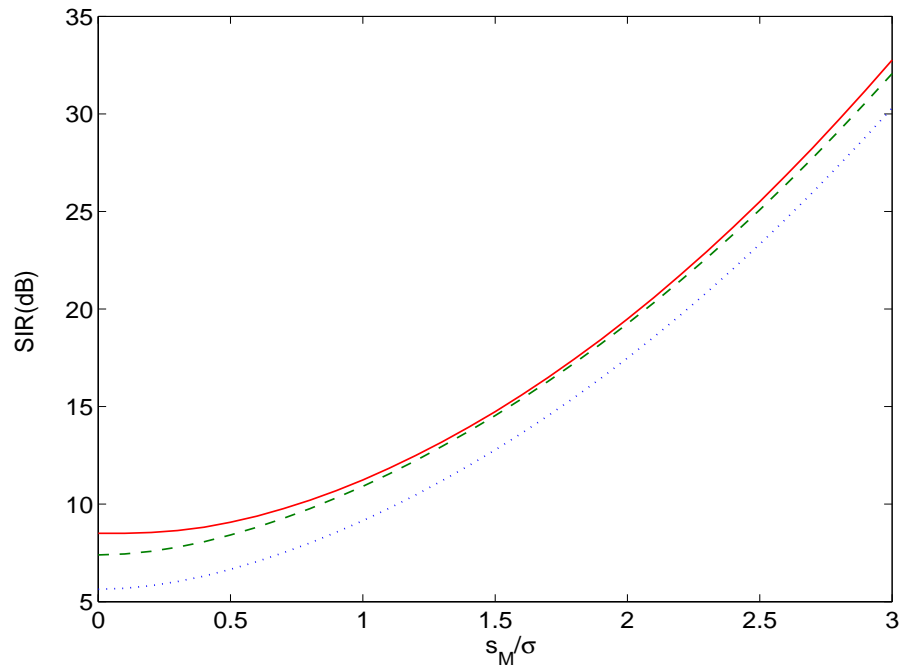

Fig. 5. $S I R_{T x, I B}$ when $M_{T x}=1$ (dotted line) or 2 (solid line) and $3 S I R_{N L} / 2$ (dashed line).

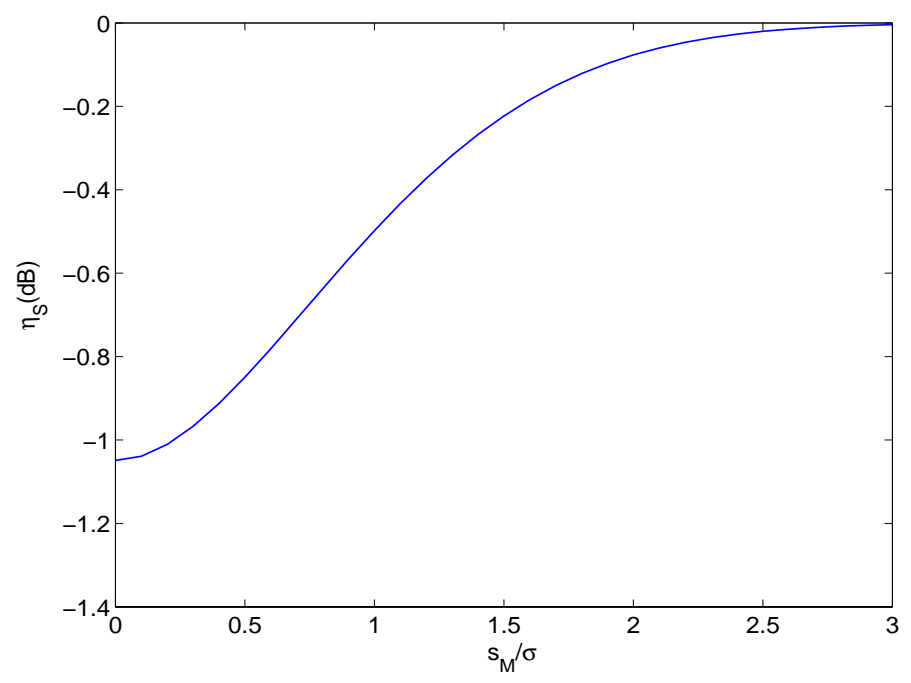

Fig. 6. Evolution of $\eta_{S}$.

Let us first consider a conventional receiver structure and an ideal AWGN channel. The theoretical BER performances, together with the corresponding simulated results, are depicted in Figs. 7 and 8. We have $U / K=1$, corresponding to a fully loaded system, in Fig. 7 and $U / K=1 / 2$ in Fig. 8. Clearly, our analytical approach is very accurate. The approximate BER formulas are slightly pessimistic for low clipping levels; for moderate and high clipping levels they are very accurate (this is in accordance with Fig. 5). It should be noted that the increase in the robustness against nonlinear effects when $U / K<1$ (implicit in the formulas of Sec. IV) was confirmed by the simulations. It should also be noted that similar results were observed for other values of $N$, provided that $N$ is high enough 


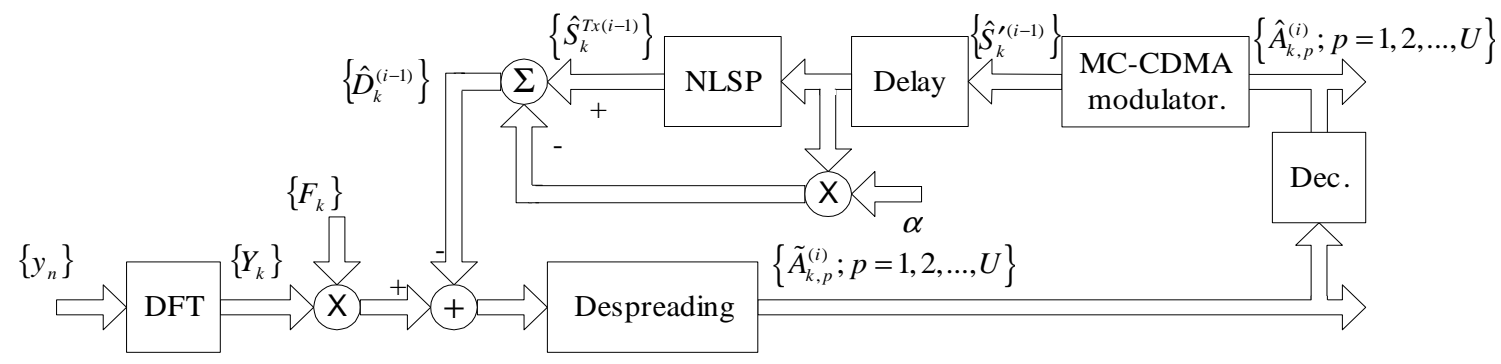

Fig. 4. Enhanced receiver structure with iterative cancellation of nonlinear distortion effects (NLSP: nonlinear signal processing chain similar to that of Fig. 2).

to validate the Gaussian approximation for the signals at the input of the nonlinear device (in fact, our analytical approach is already very accurate with a few tens of subcarriers, at least for $\left.\mathrm{BER} \geq 10^{-4}\right)$.

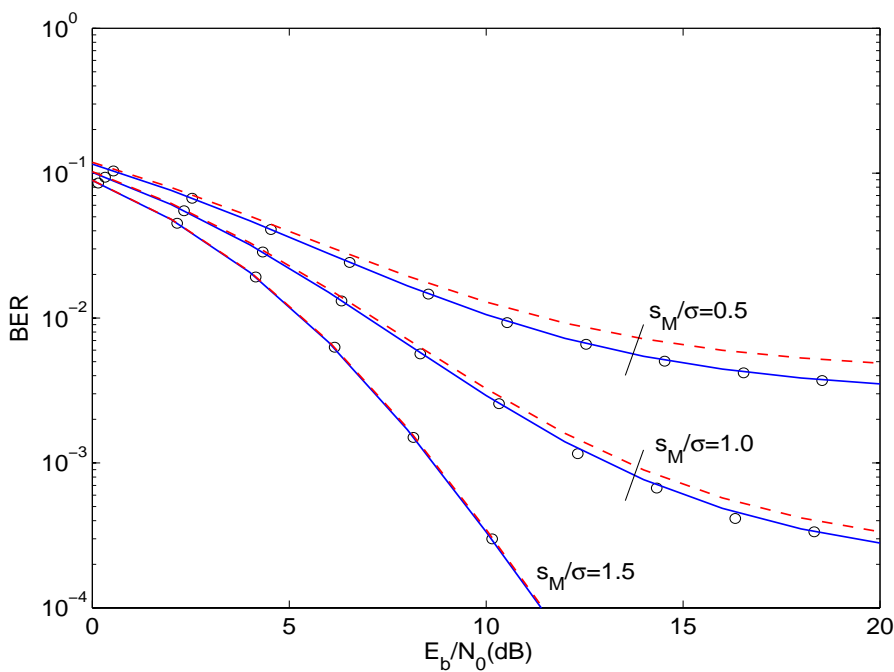

Fig. 7. Exact (solid line) and approximate (dashed line) BER formulas when $U / K=1$ (the (o) denote the simulated results)

Let us consider now the enhanced receiver structure depicted in Fig. 4, where an iterative estimation and cancelation of the nonlinear self-interference component is performed. The corresponding performances for an ideal AWGN channel are depicted in Figs. 9 and 10. Clearly, the iterative receiver has excellent performances, being able to remove almost entirely the self-interference component, at least for moderate and high values of $E_{b} / N_{0}$, even for small clipping levels.

Let us consider a time-dispersive channel. As an example, we adopted the HIPERLAN/2 channel C [22] (similar results were obtained for other time-dispersive channels). The useful part of the block has duration $T=4 \mu \mathrm{s}$ and the cyclic extension has duration $T_{G}=1.1 \mu \mathrm{s}$ (the duration of the channel impulse response is $1.05 \mu \mathrm{s}$ ), corresponding to MC-CDMA blocks with duration $T_{B}=T+T_{G}=5.1 \mu \mathrm{s}$. The channel

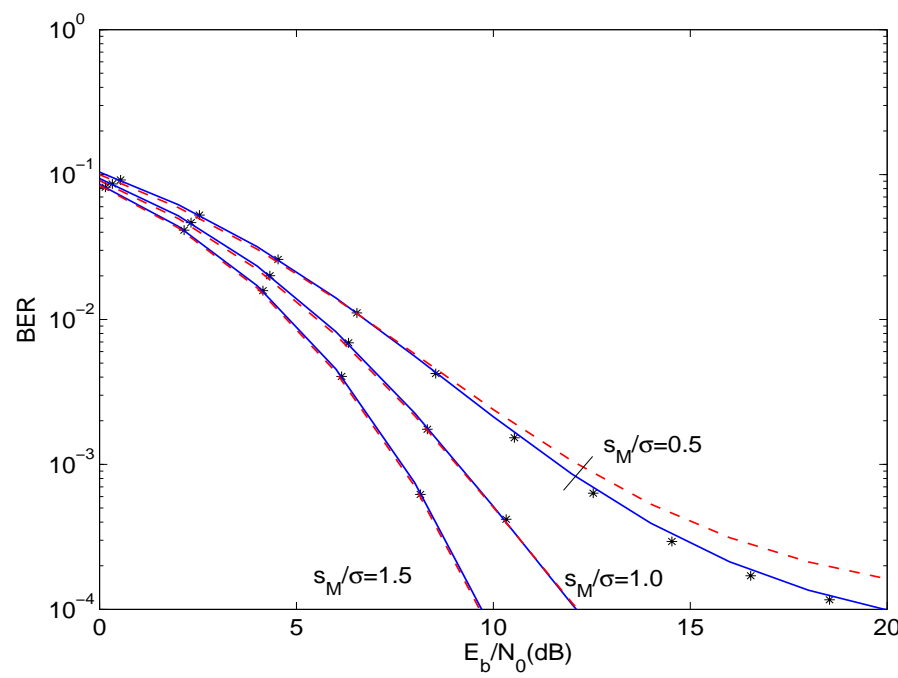

Fig. 8. Exact (solid line) and approximate (dashed line) BER formulas when $U / K=1 / 2$ (the ${ }^{*}$ ) denote the simulated results).

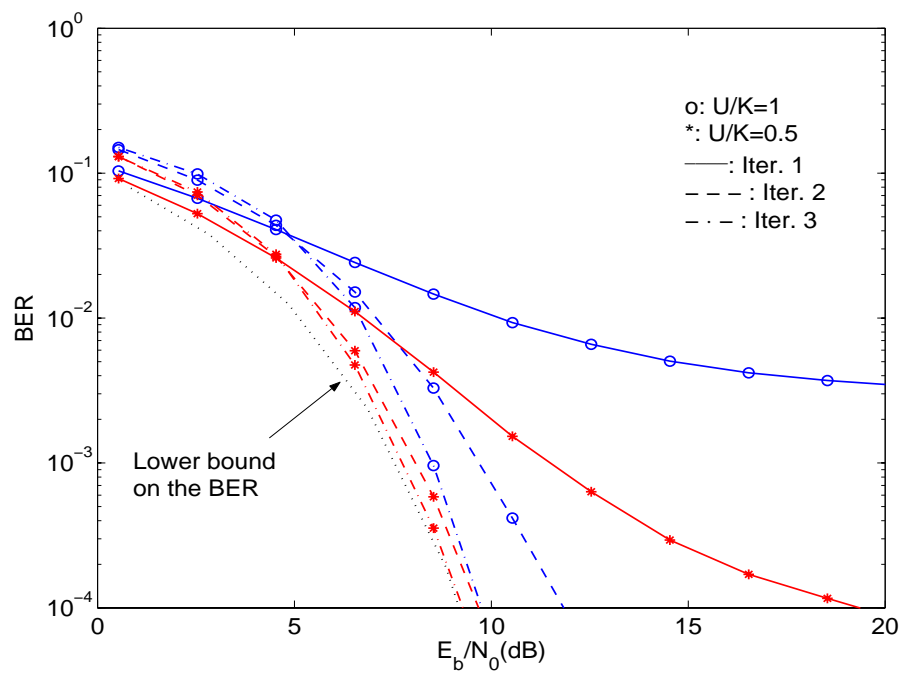

Fig. 9. BER performance for the iterative receiver when $s_{M} / \sigma=0.5$. 


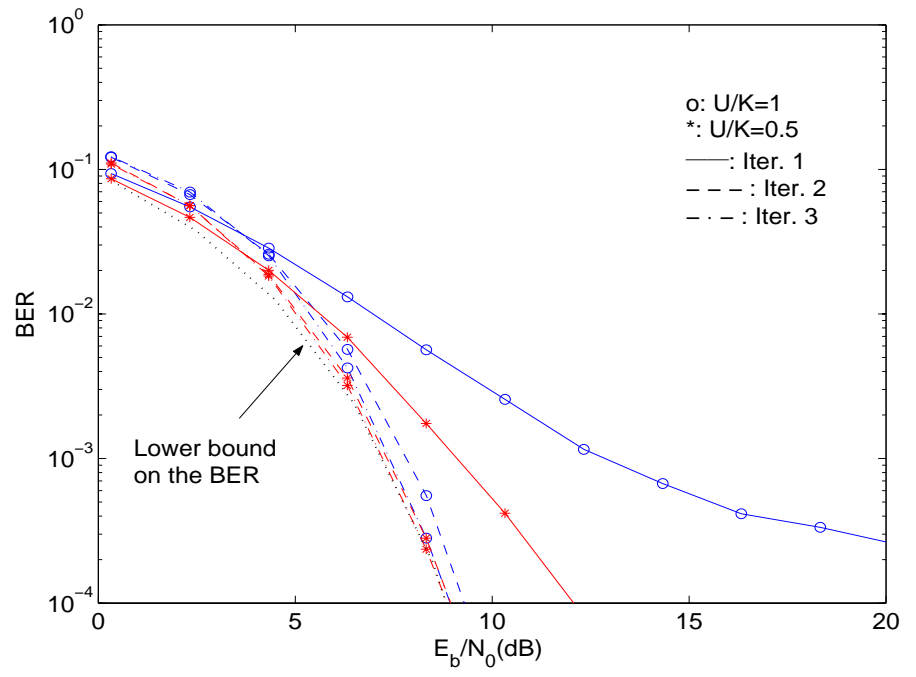

Fig. 10. BER performance for the iterative receiver when $s_{M} / \sigma=1.0$.

is invariant for the duration of each block and we assumed perfect channel estimation at the receiver. Fig. 11 presents the performance of the iterative receiver. Clearly, the iterative receiver structure is still able to cancel almost entirely the nonlinear self-interference component.

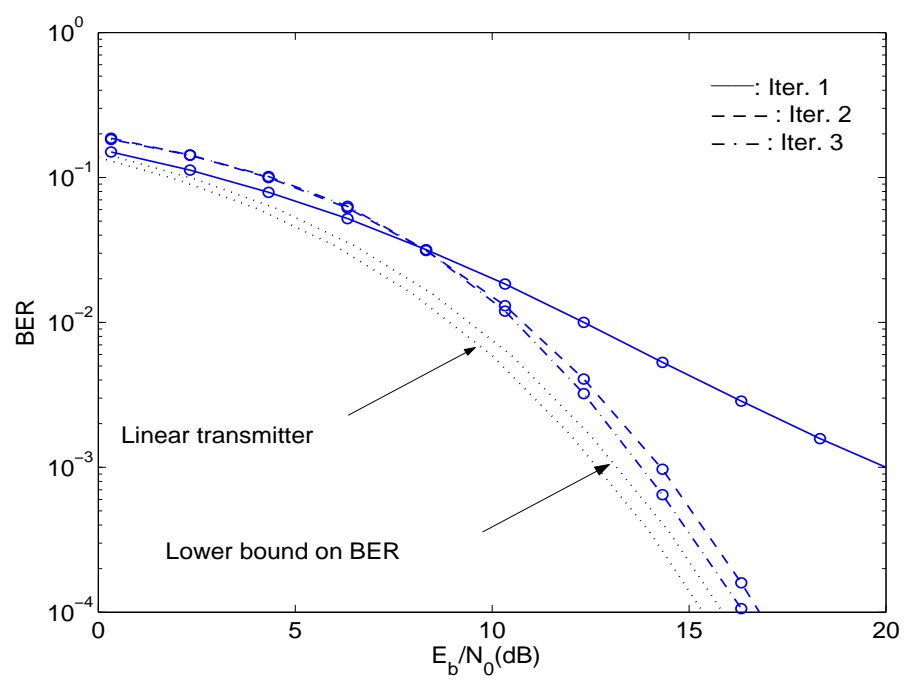

Fig. 11. BER performance for the iterative receiver when $s_{M} / \sigma=1.0$, $U / K=1$ and a time-dispersive channel.

\section{Conclusions and Final Remarks}

In this paper we presented an analytical tool for the performance evaluation of nonlinear effects in MC-CDMA signals. For this purpose, we took advantage of the Gaussianlike behavior of MC-CDMA signals with a large number of subcarriers and employed well-known results on memoryless nonlinear devices with Gaussian inputs to statistically characterize the signals at the output of the nonlinear device. We also included analytical, exact formulas for the BER computation, as well as low complexity, approximate formulas which require only the evaluation of two integrals (if the nonlinear device corresponds to an ideal envelope clipping, these two integrals can be written in closed form). Since the OFDM signals can be regarded as MC-CDMA signals with $K=1$ and $M=N$, our low-complexity SIR and BER expressions can also be used for evaluating the nonlinear effects in OFDM schemes.

It was shown that the ratio between the number of used channels and the spreading factor has a key influence on the robustness of a given MC-CDMA scheme to nonlinear effects. The higher this ratio the lower the robustness to nonlinear effects. The spreading provides a diversity effect over the nonlinear interference.

It should be noted that, by using our statistical characterization of the signals at the output of the nonlinear device we can simplify Monte-Carlo simulations: due to the Gaussian nature of the nonlinear self-interference component, we do not need to simulate the nonlinear operation (we just need to modify the noise variance to include both the channel noise and the self-interference component).

Our simplified analytical approach could also be employed for the performance evaluation of conventional OFDM schemes in the presence of the same nonlinear distortion effects (in fact, the OFDM schemes can be regarded as MC-CDMA schemes with spreading factor $K=1$ and $M=N$ ).

\section{Appendix A.}

Let us assume an infinite oversampling factor $\left(M_{T x}=\right.$ $+\infty)$. If all subcarriers have the same average power and its number is very high then the autocorrelation of the complex envelope of the signal at the input of the nonlinear device, referred to the central frequency of the spectrum, is approximately given by $R_{i n}(\tau)=P_{i n} \operatorname{sinc}(\tau B)$, with $P_{i n}$ denoting its power and $B$ its bandwidth. The corresponding PSD is $G_{i n}(f)=P_{i n} \operatorname{rect}(f / B) / B$.

If we assume that the power of the nonlinear selfinterference power, $P_{N L}^{I}$, is associated to the IMP of order 3 (i.e., $P_{3}=P_{N L}^{I}$ and $P_{2 \gamma+1}=0, \gamma>1$ ), then the autocorrelation of the self-interference component is (see (11))

$$
R_{d}(\tau)=2 P_{3}=2 P_{N L}^{I} \frac{R_{i n}^{3}(\tau)}{R_{i n}^{3}(0)},
$$

and the corresponding PSD is

$$
\begin{array}{r}
G_{d}(f)=\mathcal{F}\left\{R_{d}(\tau)\right\}= \\
=2 P_{N L}^{I} \frac{G_{i n}(f) * G_{i n}(f) * G_{i n}(f)}{R_{i n}^{3}(0)}=\frac{2 P_{N L}^{I} G_{3}(f / B)}{B}
\end{array}
$$

where $G_{3}(f)=G_{1}(f) * G_{1}(f) * G_{1}(f)$, with $G_{1}(f)=\operatorname{rect}(f)$. Clearly,

$$
G_{3}(f)= \begin{cases}3 / 4-f^{2}, & |f| \leq 1 / 2 \\ 9 / 8-3|f| / 2+f^{2} / 2, & 1 / 2<|f|<3 / 2 \\ 0, & |f| \geq 3 / 2\end{cases}
$$

Therefore, the total in-band power is $P_{N L, I B}^{I}=$ $\int_{-B / 2}^{B / 2} G_{d}(f) d f$. For $1 \geq M_{T x} \geq 2$, we have aliasing 
effects in the in-band region. Therefore, the total in-band power is

$$
P_{N L, I B}^{I}=\int_{-B / 2}^{B / 2}\left(G_{d}(f)+G_{d}(f-B)+G_{d}(f+B)\right) d f .
$$

It can be shown that, in this case,

$$
P_{N L, I B}^{I}=\kappa\left(M_{T x}\right) P_{N L}^{I},
$$

with

$\kappa\left(M_{T x}\right)= \begin{cases}\frac{1}{3}\left(-M_{T x}^{3}+6 M_{T x}^{2}-12 M_{T x}+10\right), & 1 \leq M_{T x}<2 \\ \frac{2}{3}, & M_{T x} \geq 2 .\end{cases}$

\section{References}

[1] N. Yee, J. Linnartz, and G. Fettweis, "Multi-Carrier CDMA in Indoor Wireless Radio", IEEE PIMRC'93, Yokohama, Japan, Dec. 1993.

[2] A. Chouly, A. Brajal, and S. Jourdan, "Orthogonal Multicarrier Techniques Applied to Direct Sequence Spread Spectrum CDMA Systems”, IEEE GLOBECOM'93, Houston, TX, Nov. 1993.

[3] S. Hara and R. Prasad, "Overview of Multicarrier CDMA", IEEE Comm. Magazine, Dec. 1997.

[4] S. Hara and R. Prasad, "Design and Performance of Multicarrier CDMA System in Frequency-Selective Rayleigh Fading Channels", IEEE Trans. on Vehicular Technology, Vol. 48, No. 5, Sep. 1999

[5] L. Cimini Jr., "Analysis and Simulation of a Digital Mobile Channel using Orthogonal Frequency Division Multiplexing", IEEE Trans. on Comm., Vol. 33, No. 7, July 1985.

[6] X. Li and L. Cimini, "Effects of Clipping and Filtering on the Performance of OFDM", IEEE Comm. Letters, May 1998.

[7] R. Dinis and A. Gusmão, "A Class of Signal Processing Algorithms for Good Power/Bandwidth Tradeoffs with OFDM Transmission", IEEE ISIT 2000, Sorrento, Italy, June 2000.

[8] R. Dinis and A. Gusmão, "Signal Processing Schemes for Power/Bandwidth Efficient OFDM Transmission with Conventional or LINC Transmitter Structures", IEEE ICC'01, Helsinki, June 2001.

[9] R. Dinis and A. Gusmão, "A Class of Nonlinear Signal Processing Schemes for Bandwidth-Efficient OFDM Transmission with Low Envelope Fluctuation", IEEE Trans. on Comm., Vol. 52, No. 11, pp. 2009-2018, Nov. 2004.

[10] A. Brajal and A. Chouly, "Compensation of Nonlinear Distortions for Othogonal Multicarrier Schemes Using Predistortion", IEEE GLOBECOM'94, San Francisco, CA, Nov. 1994.

[11] A. Saleh, "Frequency-Independent and Frequency-Dependent Nonlinear Models of TWT Amplifiers", IEEE Trans. on Comm.,

[12] Rol. Dinis and Aov. Gusmá

12] R. Dinıs and A Gusmão, "Performance Evaluation of OFDM Transmission with Conventional and Two-Branch Combining Power Amplification Schemes", IEEE GLOBECOM'96, Nov.

[13] 1996. Banelli and S. Cacopardi, "Theoretical Analysis and Performance of OFDM Signals in Nonlinear AWGN Channels", IEEE Trans. on Comm., Vol. 48, Mar. 2000.

[14] R. Dinis and A. Gusmão, "On the Performance Evaluation of OFDM Transmission Using Clipping Techniques", IEEE VTC'99 (Fall), Amsterdam, Sep. 1999.
[15] J. Tellado, L. Hoo and J. Cioffi, "Maximum Likelihood Detection of Nonlinearly Distorted Multicarrier Symbols by Iterative Decoding”, IEEE Trans. on Comm. Vol. 51, Feb. 2003.

[16] A.Gusmão and R.Dinis, "Iterative Receiver Techniques for Cancellation of Deliberate Nonlinear Distortion in OFDM-type Transmission”, Int. OFDM Workshop'04, Dresden, Sep. 2004.

[17] T. Ojamperä and R. Prasad, Wideband CDMA for Third Generation Mobile Communications, Artech House Publ., 1998.

[18] H. Sari, "Orthogonal Multicarrier CDMA and its Detection on Frequency-Selective Channels", European Trans. on Telecomm., Vol. 13, No. 5, pp. 439-445, Sep.-Oct. 2002.

[19] H.Rowe, "Memoryless Nonlinearities with Gaussian Input: Elementary Results", Bell System Tech. Journal, Vol. 61, Sep. 1982.

[20] G. Stette. "Calculation of Intermodulation from a Single Carrier Amplitude Characteristic", IEEE Trans. on Comm., Vol. 22, No. 3, pp. 319-323, Mar. 1974.

[21] M. Abramowitz and I. Stegun. Handbook of Mathematical Functions, New York, Dover Publications, 1972.

[22] ETSI, "Channel models for HIPERLAN/2 in Different Indoor Scenarios", ETSI EP BRAN 3ERIO85B, pp. 1-8, March 1998. 